DOI: 10.1002/anie.200502862

\section{Label-Free Affinity Assays by Rapid Detection of Immune Complexes in Submicrometer Pores**}

\author{
Jeffrey D. Uram, Kevin Ke, Alan J. Hunt, and \\ Michael Mayer*
}

We present herein a method that uses a submicrometer pore to detect and characterize immune complexes consisting of proteins such as staphylococcal enterotoxin B (an agent with bioterrorism potential) and polyclonal antibodies. The assay is rapid, label free, requires no immobilization or modification of the antibody or antigen, and achieves single-aggregate sensitivity by monitoring changes in electrical resistance when immune complexes pass through the submicrometer pore. Adopting a recently developed nanofabrication technique based on a femtosecond-pulsed laser made it possible to fabricate pores with conical geometries and diameters as small as $575 \mathrm{~nm}$. These pores allowed sensing of immune complexes that consisted of 610-17300 proteins and detection of proteins at concentrations as low as $30 \mathrm{~nm}$. Monitoring the passage of individual immune complexes enabled determination of the size distribution and the growth of these complexes. This method senses immune complexes (and potentially other molecules or nanoparticles that can be induced to form specific assemblies) in solution, and the antibody or antigen to be detected can be present in complex media such as serum. Owing to the small footprint and simple detection scheme, submicrometer pore-based sensing of specific complexes may enable portable or high-throughput immunoassays for diagnostics and biodefense.

Coulter counting, which monitors the transient change in resistance (resistive pulse) that occurs when a particle passes

[*] Prof. M. Mayer

Departments of Biomedical Engineering and Chemical Engineering University of Michigan

Engineering Research Building (ERB), Room 4107

2200 Bonisteel Blvd., Ann Arbor, MI 48109-2099 (USA)

Fax: (+1) 734-763-4371

E-mail:mimayer@umich.edu

J. D. Uram, K. Ke

Department of Biomedical Engineering

University of Michigan

Ann Arbor, MI 48109-2099 (USA)

Prof. A. J. Hunt

Department of Biomedical Engineering and Center for Ultrafast

Optical Science

University of Michigan

Ann Arbor, MI 48109-2099 (USA)

[**] This work was supported by an NSF CAREER award (M.M.) and by a research grant from IMRA America and AISIN USA as well as seed funds from the College of Engineering, University of Michigan. The authors thank Daniel J. Estes, Amy P. Wong, Irina Gitlin, and Paul Bina for valuable discussions.

(2) Supporting information for this article is available on the WWW under http://www.angewandte.org or from the author. through a small pore filled with electrolyte, is a technique for detecting and analyzing micro-, and increasingly, nanoscale objects. As the sensitivity of a Coulter counter increases with decreasing pore diameter and length, ${ }^{[1]}$ numerous techniques have been developed for the fabrication of single-nanopore $^{[2-10]}$ or nanotube membranes. ${ }^{[1,11-13]}$ Pore-forming proteins in planar lipid bilayers (PLBs) have been elegantly used as versatile nanopore sensors ${ }^{[14-20]}$ fabricated structures, in comparison, can offer a high degree of robustness and withstand environmental stress such as vibration, pressure, extreme $\mathrm{pH}$, and elevated temperatures. Fabricated nanopores and nanotubes have been used for resistive-pulse sensing to detect viruses, ${ }^{[1]}$ the aggregation of colloids, ${ }^{[2]}$ DNA,${ }^{[4,7-11]}$ nanoparticles, ${ }^{[1,13,23,24]}$ and proteins. ${ }^{[12,25]}$ The two reports of protein detection relied either on immobilized molecular-recognition agents on the walls of the nanopore ${ }^{[25]}$ or on functionalized colloids. ${ }^{[12]}$ We hypothesized that a specific protein could be detected rapidly, without the need for immobilization or labeling, by combining a submicrometer pore with Coulter counting to monitor the formation of immune complexes in solution.

We adopted and optimized a recently developed nanomachining technique that employs femtosecond-pulsed lasers $^{[26-28]}$ to fabricate submicrometer pore structures in borosilicate glass coverslides (see Figure 1 and the Supporting Information). This technique has the advantage that it does not require masks, etching, or high vacuum and that it can fabricate in glass. Glass is an excellent substrate material

a)

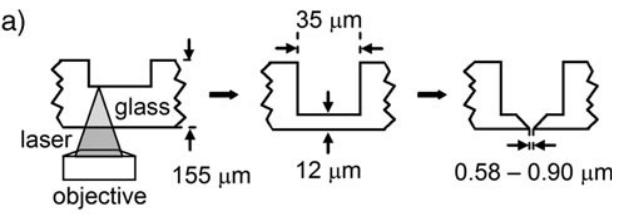

b)
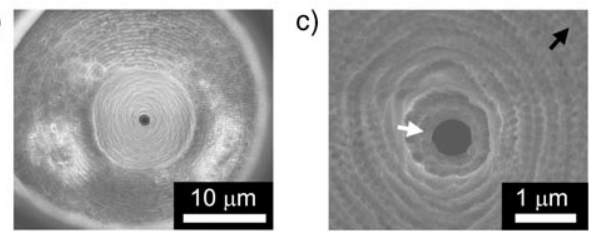

Figure 1. Laser-based fabrication of submicrometer pores with conical geometry. a) Femtosecond-pulsed lasers enabled nanomachining of conical pores in glass with diameters as small as $575 \mathrm{~nm}$; b) Scanning electron microscope (SEM) image looking into the $35-\mu \mathrm{m}$ cylinder of a pore (see a); c) SEM image focused on the narrowest part of the pore (diameter: $650 \mathrm{~nm}$ ). The conical shape of the pores was confirmed by observing different focal planes with SEM (white arrow: in focus, black arrow: out of focus).

owing to its low-noise properties, ${ }^{[29]}$ its chemical and mechanical robustness, and its amenability to surface functionalization. Furthermore, laser nanomachining is able to fabricate complicated 3D structures in optically transparent substrates. ${ }^{[30]}$ This enabled us to generate pores with a conical geometry and diameters of 575, 650 (Figure 1b,c), and $900 \mathrm{~nm}$ (see the Supporting Information). The conical shape made it possible to produce low-resistance pores in thick ( $>$ $1 \mu \mathrm{m})$ membranes that have low electrical capacitance. ${ }^{[31]}$ 
Decreasing the resistance increases the amplitude of resistive pulses as well as the rate of transport through the pore for a given pore diameter ${ }^{[7,24]}$ Lowering the capacitance can reduce electrical-current noise ${ }^{[31,32]}$ which permits recording at high bandwidths ${ }^{[31]}$ and increases the sensitivity of the Coulter counter. ${ }^{[13]}$

We mounted the glass slide with the pores onto a fluidic setup (Figure 2) made of poly(dimethylsiloxane) (PDMS) to

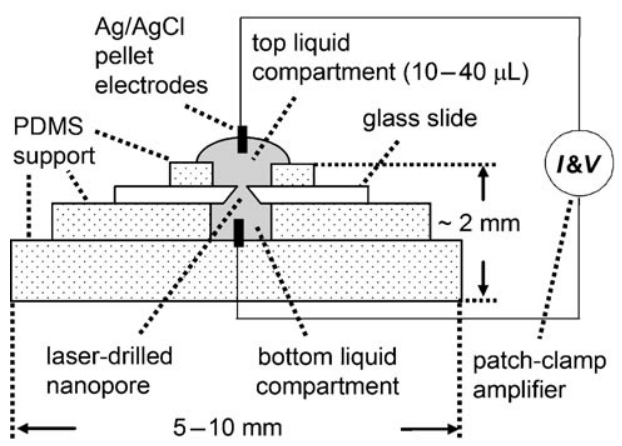

Figure 2. Side view of the experimental setup. A patch-clamp amplifier applies a constant voltage and detects small changes in current (pA-range) with fast time resolution $(10-50 \mathrm{kHz})$. A poly(dimethylsiloxane) (PDMS) fluidic setup allows for replacement of solution on either side of the submicrometer pore.

characterize their electrical properties and to perform affinity assays. The composition of the recording buffer, the resulting electrical resistances, and noise values of the pores are listed in the Supporting Information. Before studying immune complexes, we characterized the response of the nano-Coulter counter by using synthetic nanoparticles. In a cylindrical pore, the resistive pulse from a spherical particle is proportional to the volume of the particle (as long as the particle diameter is less than $\approx 0.4$ of the diameter of the pore). ${ }^{[1]}$ When we passed particles with diameters of 100,130 , and $160 \mathrm{~nm}$ through a conical pore, we observed a linear relationship between the amplitude of the current peak and the particle volume (see the Supporting Information). This linear relationship, in conjunction with evidence that particles of the same volume but varying shape give rise to resistive pulses with similar amplitudes, ${ }^{[33]}$ made it possible to estimate the volume of the immune complexes and consequently the number of proteins in a complex (see the Supporting Information).

To use the submicrometer pores to detect and characterize immune complexes, we monitored the resistive pulses that occurred when these complexes passed through the pore. The antibody-antigen model system investigated herein consisted of a goat anti-mouse antibody and a mouse monoclonal anti-baculovirus antibody as the antigen (see the Supporting Information). We examined three different equimolar concentrations $(15,30$, and $151 \mathrm{~nm})$ of the antigen and the anti-mouse antibody by using a pore with a diameter of $650 \mathrm{~nm}$. The assay was able to detect immune complexes at a concentration of $151 \mathrm{~nm}$ and $30 \mathrm{~nm}$ as shown by the resistive pulses in Figure $3 \mathrm{~b}$, c; we did not detect any immune complexes at a concentration of $15 \mathrm{~nm}$ of antibody and antigen.

Figure $3 \mathrm{~b}, \mathrm{c}$ shows that the amplitudes of many resistive pulses, caused by the immune complexes formed at a concentration of $151 \mathrm{nM}$, were considerably larger than those formed at $30 \mathrm{~nm}$. This result indicates that the immune complexes grew larger at $151 \mathrm{~nm}$ than they did at $30 \mathrm{~nm}$, and may explain why no immune complexes could be detected at a concentration of $15 \mathrm{~nm}$. Indeed, the immune complexes that formed at a concentration of $151 \mathrm{~nm}$ grew so large that they eventually blocked the pore (Figure $3 \mathrm{~b}$, the arrow indicates the onset of pore blockage; see the Supporting Information).
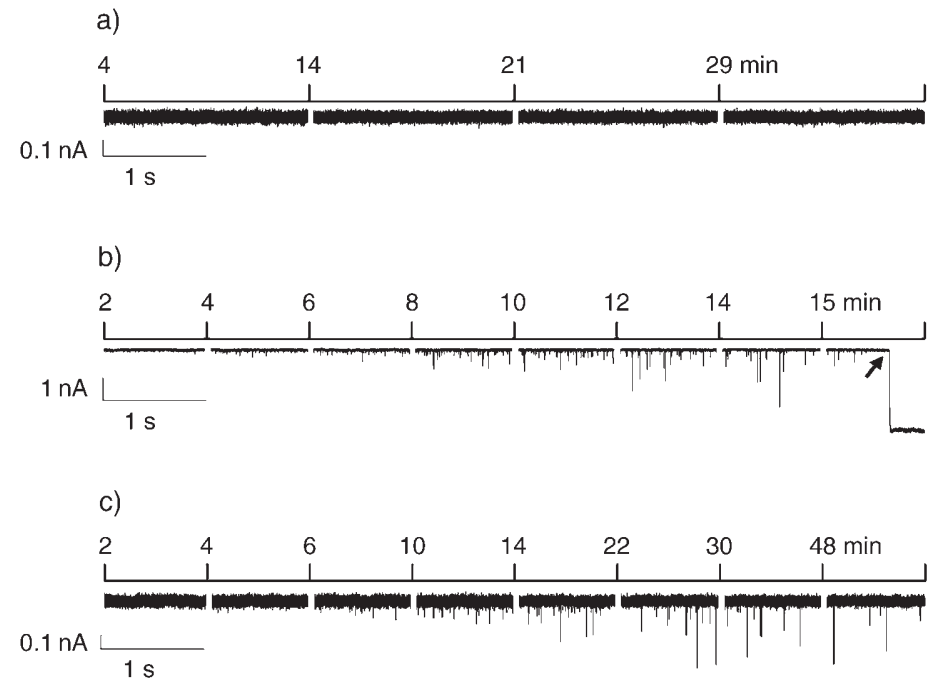

Figure 3. Time courses of the formation of immune complexes in solution. a) Control experiment with the antigen (mouse monoclonal anti-baculovirus antibody) and a nonspecific anti-rabbit antibody, both at a final concentration of $151 \mathrm{nM}$; b) At a final concentration of $151 \mathrm{~nm}$ of antigen and the specific anti-mouse antibody, detectable immune complexes formed rapidly and eventually blocked the pore (arrow). Note the $y$-scale of b) is ten times larger than the scale of the other traces owing to the large size of the immune complexes; c) At a lower antibody-antigen concentration (30 nM), detectable immune complexes formed but were smaller and did not block the pore. Each current trace is composed of multiple, short-duration recordings (length 1$2 \mathrm{~s}$; see marked scale) that were taken from data files recorded during the course of the experiment; a small gap separates each of these short recordings. The time in minutes, after the addition of anti-mouse or antirabbit antibody to the recording buffer that contained the antigen, is indicated above the beginning of each short recording. These recordings therefore represent short "snapshots" of the current activity throughout the entire experiment of several minutes duration. A pore with a diameter of $650 \mathrm{~nm}$ (Figure $1 \mathrm{~b}, \mathrm{c}$ ) was used for these experiments.

We performed a control experiment by using the same antigen and a nonspecific goat anti-rabbit antibody at a concentration of $151 \mathrm{~nm}$. We did not detect any immune complexes or pore blockage in the presence of this control antibody (Figure $3 \mathrm{a}$ ). Subsequent addition of the anti-mouse antibody at a concentration of $151 \mathrm{~nm}$ produced detectable 
immune complexes within 3 minutes, and blockage of the pore, owing to large immune complexes ("immunospecific" blockage), occurred after approximately 9 minutes. This blockage provided a dramatic response (significant and permanent change in the resistance of the submicrometer pore) that could be sensed by using simple electronics with low time resolution; it may potentially be useful for disposable, ultrasmall, and portable low-power sensors for the detection of biowarfare agents such as staphylococcal enterotoxin B (SEB) (see Figure 4), botolinum toxin, or ricin. ${ }^{[25]}$
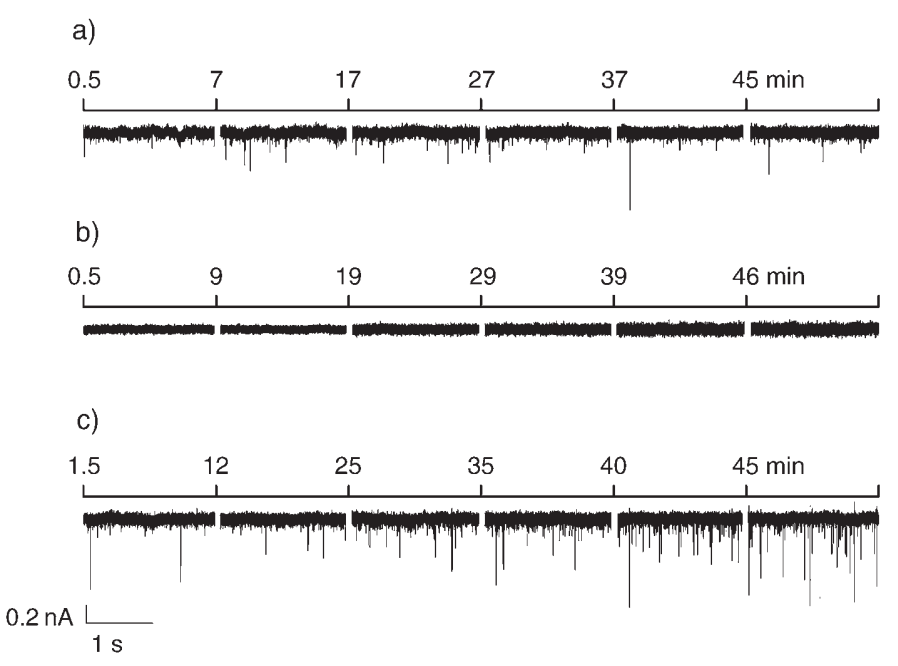

Figure 4. Detection of staphylococcal enterotoxin B (SEB) by sensing the formation of immune complexes in media containing a complex sample matrix. a) Current traces of anti-SEB serum only: one microliter of anti-SEB serum was added to $29 \mu \mathrm{L}$ of recording buffer. The nonspecific events were caused by serum components that were not removed by a membrane filter with pores of $0.1 \mu \mathrm{m}$; b) Current traces of SEB only (final concentration: $200 \mathrm{nM}$ ); c) Current traces of SEB and anti-SEB serum: SEB at a final concentration of $200 \mathrm{~nm}$ and $1 \mu \mathrm{L}$ of anti-SEB serum in a total volume of $32 \mu \mathrm{L}$. The addition of anti-SEB serum caused a significant increase in the number and size of events compared to a). Each current trace is composed of multiple, shortduration (length $2 \mathrm{~s}$, see marked scale) recordings that were taken from data files recorded at different times during the experiment; a small gap separates each recording. The time in minutes after the addition of anti-SEB serum, or SEB, to the recording buffer is indicated above the beginning of each short recording.

To test the ability of the pore-based sensor to detect proteins on a relevant system in complex media, we detected staphylococcal enterotoxin B (SEB) by using sheep anti-SEB serum. SEB is a causative agent of food poisoning and has the potential for bioterrorism according to the National Institute of Allergy and Infectious Disease of the USA. ${ }^{[34]}$ The addition of the anti-SEB serum to a solution containing SEB caused a large increase in the size and number of detectable aggregates when compared with the anti-SEB serum alone (Figure 4). We obtained similar results with a second system that employed rabbit antiserum to detect a monoclonal antibody (see the Supporting Information). These results demonstrate that submicrometer pore-based sensors can detect immune complexes in media that contain complex samples such as blood serum.
In addition to detecting immune complexes, and hence antigens or antibodies, submicrometer pore-based Coulter counting offers the possibility to evaluate specific properties of these complexes, such as their volume and growth rate. These properties are important as the size of an immune complex influences its physiological properties, for instance its clearance from circulation and its adherence to phagocytes. ${ }^{[35]}$ Studying polydisperse immune complexes is difficult owing to their large heterogeneity. Light-scattering techniques have been used $;^{[35,36]}$ however, as they measure multiple particles at once, these techniques can be problematic for characterizing polydisperse samples. ${ }^{[37]}$ In contrast, Coulter counting measures each particle individually and therefore can provide information on the volume, polydispersity, and growth of the immune complexes with single-aggregate sensitivity.

To demonstrate these capabilities, we monitored the increase in volume of immune complexes over time (Figure $5 \mathrm{a}, \mathrm{d})$. The general trend of the average peak amplitudes compares well with data obtained by light scattering. ${ }^{[36]}$ The sigmoidal shape in Figure $5 \mathrm{~d}$ may be a consequence of a thermodynamically stable size of the immune complexes. ${ }^{[38,39]}$ Figure $5 \mathrm{a}$, d shows that the standard deviation in the amplitude of the current peaks increased significantly during the growth of the immune complexes, therefore indicating a strong increase in the polydispersity of the complexes. Interestingly, the majority of the immune complexes sensed shortly after the addition of antibody (Figure 5b,e) had volumes that were comparable to complexes that were sensed after 8 (Figure $5 \mathrm{c}$ ) and $40 \mathrm{~min}$ (Figure $5 \mathrm{f}$ ). With increasing time, however, a fraction of the complexes reached volumes that were approximately two-times larger than that of the majority of the volumes (Figure $5 \mathrm{f}$ ). This result suggests that the later stage of growth may have been caused by collisions between slowly diffusing complexes ${ }^{[36]}$ and may explain the relatively rare formation of complexes that are significantly larger than the majority.

As a result of the linear relationship between the peak amplitude and volume of immune complexes, we were able to estimate the number of proteins in an aggregate by assuming a molecular volume of $347 \mathrm{~nm}^{3}$ for an immunoglobulin $\mathrm{G}$ antibody. ${ }^{[40]}$ The volumes of the immune complexes sensed by the pore with a diameter of $650 \mathrm{~nm}$ and an antibody antigen concentration of $151 \mathrm{~nm}$ ranged from $2.1 \times 10^{5}-6.0 \times 10^{6} \mathrm{~nm}^{3}$, which corresponds to aggregates of 610-17300 proteins.

Submicrometer pore-based detection of immune complexes offers a general, rapid, label-free, and solution-based method for the detection of any protein or particle that can be triggered to form a detectable assembly, while providing information on the volume, growth, and polydispersity of individual aggregates. The detection limit of $30 \mathrm{~nm}$ for antigens compares favorably to other label-free detection techniques such as affinity capillary electrophoresis (ACE), gel-based immunoprecipitation, and direct immunoaggregation assays based on light scattering, all of which have detection limits between 10 and $1000 \mathrm{~nm}$ depending on the technique ${ }^{[41-43]}$ In addition to its benefits for affinity assays with small footprints and reagent requirements, the technique presented herein may be particularly useful for in situ 
a)

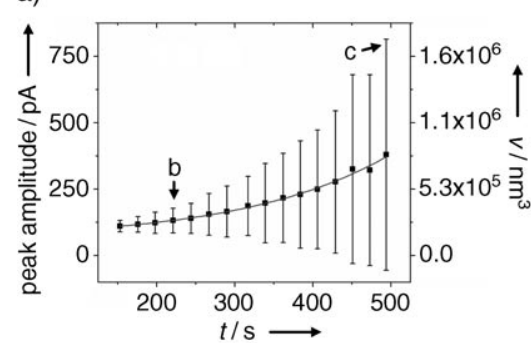

c)

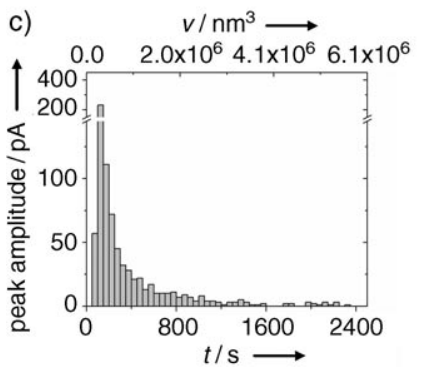

e)

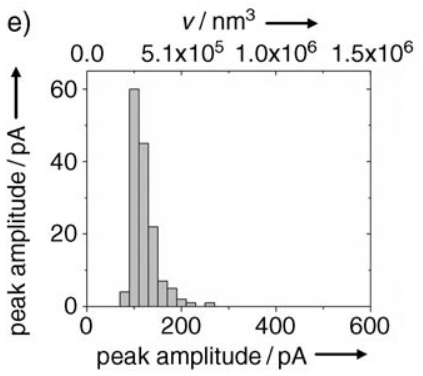

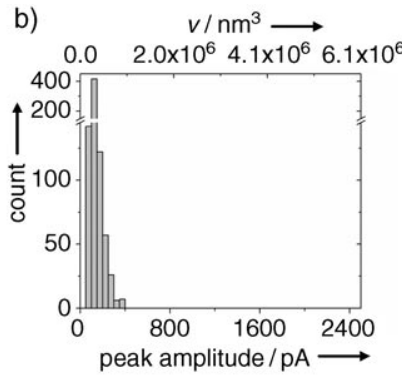

d)

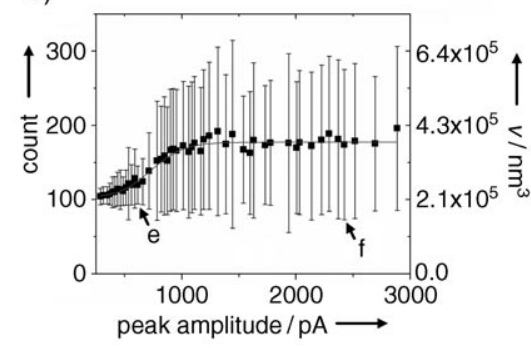

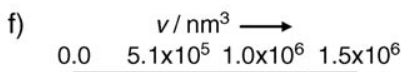

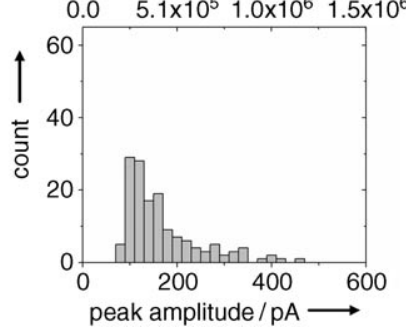

Figure 5. Time course of the current peak amplitudes and volumes of immune complexes. a) Growth of immune complexes at a concentration of $151 \mathrm{~nm}$ of both antigen (mouse monoclonal anti-baculovirus antibody) and anti-mouse antibody. A firstorder exponential function was fitted to the data. ${ }^{[3]}$ The small letters in graph a) corresponds to the time points from which the histograms shown in b) and c) were extracted; b) Peak amplitudes and volumes recorded at $240 \mathrm{~s}$ after the addition of antimouse antibody; c) Peak amplitudes and volumes recorded $490 \mathrm{~s}$ after addition of antimouse antibody. Note that $74 \%$ of the complexes maintained their volumes compared to b); however, a small fraction of complexes reached volumes that were up to ten times larger than in b); d) Growth of immune complexes at a concentration of $30 \mathrm{~nm}$. A sigmoidal function was fitted to the data. ${ }^{[6]}$ The small letters in graph d) correspond to the time points from which the histograms shown in e) and f) were estracted. e) Peak amplitudes and volumes recorded at $610 \mathrm{~s}$ after the addition of anti-mouse antibody; f) Peak amplitudes and volumes recorded $2400 \mathrm{~s}$ after the addition of anti-mouse antibody. Note the occurrence of peak amplitudes with approximately two, three, and four times the change in current $(\approx 200,300,400 \mathrm{pA})$ of those shown in e). Each point in a), d) reflects the average amplitude and aggregate volume obtained from peaks over a period of $20 \mathrm{~s}$.

monitoring of controlled assemblies of nanoparticles, ${ }^{[4-46]}$ thereby addressing an urgent need in nanotechnology. ${ }^{[47]}$

Received: August 11, 2005

Revised: December 19, 2005

Published online: February 28, 2006

Keywords: Coulter counting · immunoassays · nanomachining · nanotechnology
[1] R. W. DeBlois, C. P. Bean, Rev. Sci. Instrum. 1970, 41, 909.

[2] N. Fertig, A. Tilke, R. H. Blick, J. P. Kotthaus, J. C. Behrends, G. ten Bruggencate, Appl. Phys. Lett. 2000, 77, 1218.

[3] P. Y. Apel, Y. E. Korchev, Z. Siwy, R. Spohr, M. Yoshida, Nucl. Instrum. Methods Phys. Res. Sect. B 2001, 184, 337.

[4] J. Li, D. Stein, C. McMullan, D. Branton, M. J. Aziz, J. A. Golovchenko, Nature 2001, 412, 166.

[5] C. C. Harrell, S. B. Lee, C. R. Martin, Anal. Chem. 2003, $75,6861$.

[6] A. J. Storm, J. H. Chen, X. S. Ling, H. W. Zandbergen, C. Dekker, Nat. Mater. 2003, 2, 537.

[7] P. Chen, T. Mitsui, D. B. Farmer, J. Golovchenko, R. G. Gordon, D. Branton, Nano Lett. 2004, 4, 1333.

[8] H. Chang, F. Kosari, G. Andreadakis, M. A. Alam, G. Vasmatzis, R. Bashir, Nano Lett. 2004, 4, 1551.

[9] J. B. Heng, C. Ho, T. Kim, R. Timp, A. Aksimentiev, Y. V. Grinkova, S. Sligar, K. Schulten, G. Timp, Biophys. J. 2004, 87, 2905.

[10] A. Mara, Z. Siwy, C. Trautmann, J. Wan, F. Kamme, Nano Lett. 2004, 4, 497.

[11] O. A. Saleh, L. L. Sohn, Nano Lett. 2003, 3, 37.

[12] O. A. Saleh, L. L. Sohn, Proc. Natl. Acad. Sci. USA 2003, $100,820$.

[13] T. Ito, L. Sun, R. M. Crooks, Anal. Chem. 2003, 75, 2399.

[14] J. J. Kasianowicz, E. Brandin, D. Branton, D. W. Deamer, Proc. Natl. Acad. Sci. USA 1996, 93, 13770.

[15] L. Gu, O. Braha, S. Conlan, S. Cheley, H. Bayley, Nature 1999, 398, 686.

[16] C. Schmidt, M. Mayer, H. Vogel, Angew. Chem. 2000, 112, 3267; Angew. Chem. Int. Ed. 2000, 39, 3137.

[17] A. F. Sauer-Budge, J. A. Nyamwanda, D. K. Lubensky, D. Branton, Phys. Rev. Lett. 2003, 90, 238101.

[18] S. Howorka, J. Nam, H. Bayley, D. Kahne, Angew. Chem. 2004, 116, 860; Angew. Chem. Int. Ed. 2004, 43, 842.

[19] E. Berkane, F. Orlik, A. Charbit, C. Danelon, D. Fournier, R. Benz, M. Winterhalter, J. Nanobiotechnology 2005, 3:3.

[20] J. Sánchez-Quesada, A. Saghatelian, S. Cheley, H. Bayley, M. R. Ghadiri, Angew. Chem. Int. Ed. 2004, 43, 3063.

[21] R. W. DeBlois, E. E. Uzgiris, D. H. Cluxton, H. M. Mazzone, Anal. Biochem. 1978, 90, 273.

[22] G. K. von Schulthess, G. B. Benedek, R. W. DeBlois, Macromolecules 1983, 16, 434.

[23] O. A. Saleh, L. L. Sohn, Rev. Sci. Instrum. 2001, 72, 4449.

[24] T. Ito, L. Sun, M. A. Bevan, R. M. Crooks, Langmuir 2004, 20, 6940.

[25] Z. Siwy, L. Trofin, P. Kohli, L. A. Baker, C. Trautmann, C. R. Martin, J. Am. Chem. Soc. 2005, 127, 5000.

[26] C. B. Schaffer, A. Brodeur, J. F. García, E. Mazur, Opt. Lett. 2001, 26, 93.

[27] A. P. Joglekar, H. Liu, G. J. Spooner, E. Meyhöfer, G. Mourou, A. J. Hunt, Appl. Phys. B 2003, 77, 25.

[28] A. P. Joglekar, H. Liu, E. Meyhöfer, G. Mourou, A. J. Hunt, Proc. Natl. Acad. Sci. USA 2004, 101, 5856.

[29] J. L. Rae, R. A. Levis, Methods Enzymol. 1992, 207, 66.

[30] K. Ke, E. Hasselbrink, A. J. Hunt, Proc. SPIE-Int. Soc. Opt. Eng. 2005, 5714, 53.

[31] M. Mayer, J. K. Kriebel, M. T. Tosteson, G. M. Whitesides, Biophys. J. 2003, 85, 2687.

[32] R. A. Levis, J. L. Rae, Methods Enzymol. 1998, 293, 218.

[33] R. K. Eckhoff, J. Sci. Instrum. 1967, 44, 648.

[34] http://www.niaid.nih.gov/dmid/eid/erd.htm. 
[35] R. M. Murphy, H. Slayter, P. Schurtenberger, R. A. Chamberlin, C. K. Colton, M. L. Yarmush, Biophys. J. 1988, 54, 45.

[36] N. N. Gorgani, S. B. Easterbrook-Smith, J. G. Altin, Biochim. Biophys. Acta 1996, 1317, 45.

[37] A. Bootz, V. Vogel, D. Schubert, J. Kreuter, Eur. J. Pharm. Biopharm. 2004, 57, 369.

[38] J. Liu, P. Lester, S. Builder, S. J. Shire, Biochemistry 1995, 34, 10474.

[39] L. C. Santora, Z. Kaymakcalan, P. Sakorafas, I. S. Krull, K. Grant, Anal. Biochem. 2001, 299, 119.

[40] S. W. Schneider, J. Lärmer, R. M. Henderson, H. Oberleithner Pflügers Arch. 1998, 435, 362.

[41] R. M. Guijt-van Duijn, J. Frank, G. W. K. van Dedem, E. Baltussen, Electrophoresis 2000, 21, 3905.

[42] I. Roitt, J. Brostoff, D. Male, Immunology, 6th ed., Mosby, Edinburgh, UK, 2001.

[43] C. P. Price, D. J. Newman, in Principles and Practice of Immunoassay, 2nd ed., Stockton Press, New York, NY, 1997, 443.

[44] P. Hazarika, B. Ceyhan, C. M. Niemeyer, Angew. Chem. 2004, 116, 66631; Angew. Chem. Int. Ed. 2004, 43, 6469.

[45] Z. Tang, N. A. Kotov, Adv. Mater. 2005, 17, 951.

[46] J. Yang, M. Mayer, J. K. Kriebel, P. Garstecki, G. M. Whitesides, Angew. Chem. 2004, 116, 1581; Angew. Chem. Int. Ed. 2004, 43, 1555.

[47] S. C. Glotzer, M. J. Solomon, N. A. Kotov, AIChE J. 2004, 50, 2978. 